\title{
Establishment of an in vitro model for analyzing mitochondrial ultrastructure in PRKN-mutated patient iPSC-derived dopaminergic neurons
}

\author{
Mutsumi Yokota' ${ }^{1}$, Soichiro Kakuta ${ }^{2,3}$, Takahiro Shiga ${ }^{4}$, Kei-ichi Ishikawa ${ }^{4,5}$, Hideyuki Okano ${ }^{6}$, Nobutaka Hattori ${ }^{5,7}$,
} Wado Akamatsu ${ }^{4,7}$ and Masato Koike ${ }^{1,7^{*}}$

\begin{abstract}
Mitochondrial structural changes are associated with the regulation of mitochondrial function, apoptosis, and neurodegenerative diseases. PRKN is known to be involved with various mechanisms of mitochondrial quality control including mitochondrial structural changes. Parkinson's disease (PD) with PRKN mutations is characterized by the preferential degeneration of dopaminergic neurons in the substantia nigra pars compacta, which has been suggested to result from the accumulation of damaged mitochondria. However, ultrastructural changes of mitochondria specifically in dopaminergic neurons derived from iPSC have rarely been analyzed. The main reason for this would be that the dopaminergic neurons cannot be distinguished directly among a mixture of iPSC-derived differentiated cells under electron microscopy. To selectively label dopaminergic neurons and analyze mitochondrial morphology at the ultrastructural level, we generated control and PRKN-mutated patient tyrosine hydroxylase reporter (TH-GFP) induced pluripotent stem cell (iPSC) lines. Correlative light-electron microscopy analysis and live cell imaging of GFPexpressing dopaminergic neurons indicated that iPSC-derived dopaminergic neurons had smaller and less functional mitochondria than those in non-dopaminergic neurons. Furthermore, the formation of spheroid-shaped mitochondria, which was induced in control dopaminergic neurons by a mitochondrial uncoupler, was inhibited in the PRKNmutated dopaminergic neurons. These results indicate that our established TH-GFP iPSC lines are useful for characterizing mitochondrial morphology, such as spheroid-shaped mitochondria, in dopaminergic neurons among a mixture of various cell types. Our in vitro model would provide insights into the vulnerability of dopaminergic neurons and the processes leading to the preferential loss of dopaminergic neurons in patients with PRKN mutations.
\end{abstract}

Keywords: Mitochondria, Ultrastructure, PRKN, IPSC, Dopaminergic neurons

\footnotetext{
${ }^{*}$ Correspondence: mkoike@juntendo.ac.jp

1 Department of Cell Biology and Neuroscience, Juntendo University

Graduate School of Medicine, 2-1-1 Hongo, Bunkyo-ku, Tokyo 113-8421, Japan

Full list of author information is available at the end of the article
}

\begin{abstract}
Introduction
PRKN/PARK2, which encodes Parkin RBR E3 ubiquitin protein ligase, is one of the most common genes responsible for early-onset familial Parkinson's disease (PD). Parkin regulates mitochondrial quality control, such as mitochondrial fission/fusion, transport, biogenesis, and mitophagy [1-3]. The accumulation of damaged mitochondria caused by Parkin dysfunction has been suggested to contribute to pathogenesis in PD [4,
\end{abstract} permits use, sharing, adaptation, distribution and reproduction in any medium or format, as long as you give appropriate credit to the original author(s) and the source, provide a link to the Creative Commons licence, and indicate if changes were made. The images or other third party material in this article are included in the article's Creative Commons licence, unless indicated otherwise in a credit line to the material. If material is not included in the article's Creative Commons licence and your intended use is not permitted by statutory regulation or exceeds the permitted use, you will need to obtain permission directly from the copyright holder. To view a copy of this licence, visit http://creativecommons.org/licenses/by/4.0/. The Creative Commons Public Domain Dedication waiver (http://creativeco mmons.org/publicdomain/zero/1.0/) applies to the data made available in this article, unless otherwise stated in a credit line to the data. 
5]. To elucidate the detailed mechanisms of PD development, Parkin knockout mouse models have been generated in previous studies [6-10]. Nevertheless, these Parkin knockout mice have reproduced only some parts of the PD phenotypes $[6-9,11]$. In contrast, in patientspecific induced pluripotent stem cell (iPSC)-derived neurons, PRKN mutations have been reported to result in abnormal mitochondrial morphology and a failure of mitochondrial degradation [12-14], an inability to form complex neuronal morphology [15], and reduced dopamine use [16].

It has recently been reported that Parkin is associated with various types of mitochondrial structural changes, for example spheroid-shaped mitochondria [17, 18], mitochondrial-derived vesicles $[19,20]$, and the endoplasmic reticulum (ER)-mitochondria interface [21]. These structural changes in mitochondria have been considered as mechanisms of mitochondrial quality control under mitochondrial stress. To detect such structures, ultrastructural analyses of mitochondria are essential. However, it remains unknown whether the mitochondrial structural changes are caused by mitochondrial stress in PD patients with PRKN mutations, because there is a scarcity of ultrastructural studies in iPSC-derived neurons from PD patients.

Furthermore, considering that PD with PRKN mutations is characterized by the preferential loss of dopaminergic neurons in the substantia nigra pars compacta, ultrastructural changes of mitochondria should be analyzed specifically in dopaminergic neurons derived from iPSC. However, dopaminergic neuron-specific ultrastructural analysis by conventional electron microscopy proven difficult. The main reason for this would be that the dopaminergic neurons cannot be distinguished directly among a mixture of iPSC-derived differentiated cells under electron microscopy. Although the efficiency of differentiation from iPSCs into dopaminergic neurons has been improved in recent studies, it remains at approximately $30-40 \%[13,14,22]$. It is therefore necessary to selectively label dopaminergic neurons among the mixture of dopaminergic and non-dopaminergic neurons that are derived from iPSCs. Recently, several studies have reported the successful purification of dopaminergic neurons carrying a tyrosine hydroxylase (TH; a marker for identifying dopaminergic neurons) knockin reporter, either using the genome editing technology TALEN (transcription activator-like effector nuclease) or CRISPR/Cas9 systems [23-25]. However, the generation of TH reporter iPSC lines derived from PD patients, including those with PRKN mutations, has not been reported.

To label iPSC-derived dopaminergic neurons and analyze mitochondrial morphology in labelled dopaminergic neurons at the ultrastructural level, we generated control and $P R K N$-mutated patient $\mathrm{TH}$ reporter iPSC lines using the CRISPR/Cas9 system. With the generated reporter iPSC lines, we then analyzed mitochondrial morphology in reporter-positive dopaminergic neurons under normal conditions and CCCP treatment, using correlative light-electron microscopy (CLEM). CLEM is a powerful tool that provides both the locations of target proteins and/or cells and fine structural information by taking fluorescent images and the subsequent ultrastructural analysis [26, 27].

In the current study, by combining live cell imaging and CLEM of labeled dopaminergic neurons, we revealed smaller and less functional mitochondria in dopaminergic neurons compared with non-dopaminergic neurons. Moreover, we discovered mitochondrial ultrastructural changes under CCCP treatment which occurred prior to cell death and were impaired only in PRKN-mutated dopaminergic neurons. These results indicate that our $\mathrm{TH}$ reporter iPSC lines may be useful for comparing mitochondrial ultrastructure between control and PRKN-mutated dopaminergic neurons. Our findings offer insights into the vulnerability of dopaminergic neurons and the mechanisms of PD development.

\section{Methods \\ Single guide RNA (sgRNA) vector design}

The Cas9 target sites were determined by entering the exon 12 sequence of tyrosine hydroxylase (NC_000011.10) into the CRISPR design tool (http:// crispr.mit.edu/) [28]. A Target 1 site (Fig. 1a) was selected due to the highest score among candidates given by CRISPR design tool. A Target 2 site (Fig. 1a) was selected because it was close to the stop codon in the $T H$ gene. The PCR products by forward primer including the Target 1 or 2 sites (Additional file 1: Table S1) and the Universal-reverse primer [29] were inserted into an $\mathrm{RiH}$ vector, which was a gift from Dr. Akitsu Hotta at Kyoto University (Addgene plasmid \#60601) [29].

\section{Donor vector design for knock-in experiments}

To insert a donor vector into genomic DNA by homologous recombination, $5^{\prime}$ or $3^{\prime}$ homology arms $(0.9-1.1 \mathrm{~kb})$ flanking the Cas 9 target sites were amplified using primers (Additional file 1: Table S1) from genomic DNA of the 201B7 iPSC line [30]. The PCR fragments of $5^{\prime}$ or $3^{\prime}$ homology arms were inserted into EcoRI and BamHI cutting sites, respectively, of the HR130PA-1 vector (T2AGFP-pA-loxP-EF1 $\alpha$-RFP-T2A-Puro-pA-LoxP-MCS, System Biosciences) using In-Fusion technology. 


\begin{abstract}
(See figure on next page.)
Fig. 1 Generation of control and PRKN-mutated patient TH-GFP iPSC lines via the CRISPR/Cas9 system. a Cas9 target sites in the exon 12 sequence of the human TH gene. Black, magenta, and cyan characters represent intron, exon, and UTR sequences in the human TH gene, respectively. The stop codon is shown in bold. $\mathbf{b}$ Scheme describing the insertion of the reporter cassette into the TH gene by homologous recombination. Green and magenta arrows indicate the primer pairs used for the detection of the TH-GFP and unedited alleles, respectively. c The differential interference contrast (DIC) and fluorescent images showed RFP-positive knock-in iPS colonies after 8-9 days of puromycin selection. "PRKN" represents PRKN-mutated patient. Scale bar, $500 \mu \mathrm{m}$. d PCR analysis of knock-in iPS clones with TH-GFP alleles to determine whether the clones were homozygous or heterozygous for the TH-GFP allele. The TH-GFP allele produced by cleavage at the Target 1 (T1) or Target 2 (T2) site was found as a $1.5 \mathrm{~kb}$ or $1.6 \mathrm{~kb}$ band, respectively. The unedited TH allele was found as a $1.0 \mathrm{~kb}$ band. e Sanger sequencing confirmed that the donor sequence was correctly inserted into exon 12 of the TH gene in four knock-in iPSC lines. The $1.2 \mathrm{~kb}$ PCR products by primers (black arrows) were sequenced, and the sequence of the junction of the 5' homology arm and T2A-GFP at the cleavage site (boxed area) is indicated as the electropherograms. "PRKN" represents PRKN-mutated patient
\end{abstract}

\section{Human iPSCs}

Three previously reported iPSC lines were used for the knock-in experiments. 201B7, for a control line, was kindly provided by Dr. Shinya Yamanaka at Kyoto University [30]. WD39, another control line, and PB2, a PD patient line with a PRKN mutation, were established by Dr. Hideyuki Okano at Keio University [12].

\section{Generation of TH reporter iPS clones}

The control and PRKN-mutated patient iPSC lines were cultured on plates coated with iMatrix-511 (Nippi) and expanded in StemFit AK02N medium (Ajinomoto). On day 7 after reseeding, $1 \times 10^{6}$ cells were electroporated at $125 \mathrm{~V}$ for $5 \mathrm{~ms}$ using a NEPA21 electroporator (NEPAGENE) with $5 \mu \mathrm{g}$ of Cas9 vectors (Addgene plasmid \#60599: a gift from Dr. Akitsu Hotta) [29], sgRNA vectors, and donor vectors into each iPSC line. At day 2 after electroporation, the transfected iPSCs were treated with $0.75 \mu \mathrm{g} / \mathrm{mL}$ of puromycin for 8-9 days. The puromycin-resistant and RFP-positive colonies were manually picked up for PCR screening and expansion. The colonies for expansion were dissociated using TrypLE Select (Thermo Fisher Scientific) and were then cultured with CloneR (StemCell Technologies). The knock-in clones were established following PCR screening.

\section{PCR and sequencing of TH reporter iPS clones}

The identification of knock-in clones was based on the previously reported strategy for screening a reporter iPSC line [31]. PCR primers for the detection of the THGFP allele were designed to cover the $\mathrm{TH}$ gene and the GFP sequence in the donor vector (Additional file 1: Table S1). PCR primers for the identification of homozygous or heterozygous alleles were designed to cover the sequence before and after the cas 9 cleavage sites (Additional file 1: Table S1). The junction of the $5^{\prime}$ homology arm in the $\mathrm{TH}$ gene and the T2A-GFP sequence were amplified using primers (Additional file 1: Table S1), and the PCR products $(1.2 \mathrm{kbp})$ were sequenced using the Seq reverse primer (Additional file 1: Table S1).

\section{Differentiation of the TH reporter iPS clones}

The generated reporter iPSC lines were differentiated into dopaminergic neurons using previously established the direct neurosphere converting method [14, 22, 32, 33]. For mitochondrial stress, the differentiated cells were treated with $30 \mu \mathrm{M}$ of the mitochondrial uncoupler, carbonyl cyanide m-chlorophenyl hydrazine (CCCP; SigmaAldrich) for 3.5 or $24 \mathrm{~h}$ before fixation.

\section{Immunofluorescence staining and live cell imaging}

At day 7-10 of culture after reseeding dissociated neurospheres, differentiated cells were fixed with $4 \%$ paraformaldehyde in PBS for $10 \mathrm{~min}$ and permeabilized with $0.1 \%$ Triton X-100 in PBS for $15 \mathrm{~min}$ at room temperature. Cells were then blocked with 2\% BSA in PBS for $30 \mathrm{~min}$ and stained with primary antibodies for $2 \mathrm{~h}$ at room temperature. The following antibodies were used: rabbit antiTH antibody (PelFreez; 1:400), mouse anti-GFP antibody (MBL; $1: 500)$, mouse anti- $\beta 3$ tubulin antibody (SigmaAldrich; 1:400), mouse anti-Nestin antibody (SigmaAldrich; 1:200), and rabbit cleaved caspase-3 antibody (Cell Signaling Technology; 1:400). Cells were then washed three times and stained with Alexa Fluor 488or 647-conjugated secondary antibodies (Thermo Fisher Scientific; 1:800) and DAPI (Thermo Fisher Scientific; 1:10,000) for $1 \mathrm{~h}$ at room temperature. Mitochondrial membrane potential and active mitochondrial mass for live cell imaging were detected by incubation for $30 \mathrm{~min}$ with $20 \mathrm{nM}$ tetramethylrhodamine methyl ester (TMRM) (Thermo Fisher Scientific) and 100 nM Mitotracker Deep Red (Thermo Fisher Scientific), respectively. Immunostaining or live cell images were taken using a confocal microscope (Zeiss LSM880).

\section{Flow cytometry}

To detect GFP expression in dopaminergic neurons, differentiated cells were detached from culture plates with 
a

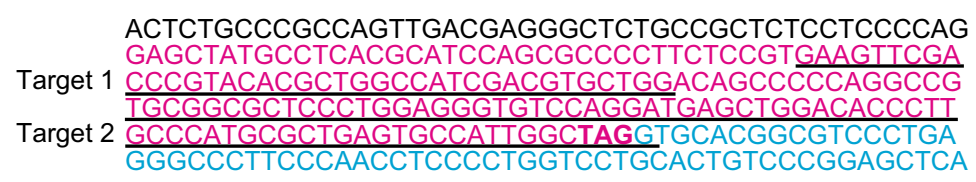

b

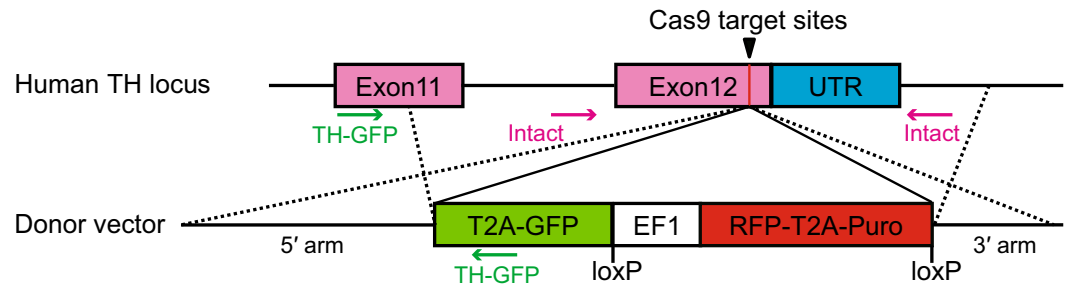

C

Control (201B7)

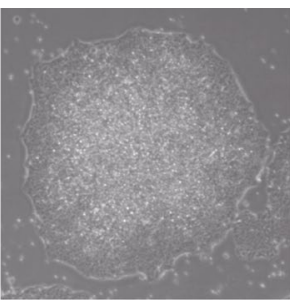

RFP

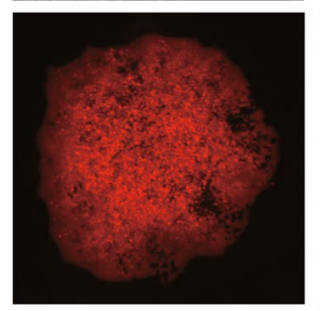

Control (WD39)
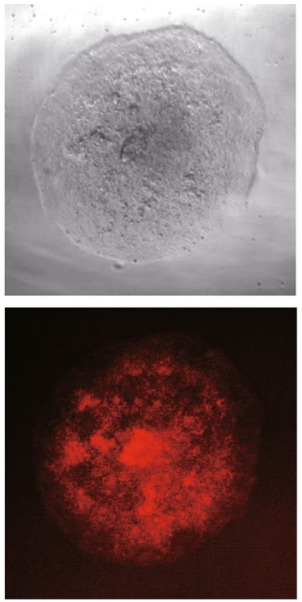

PRKN (PB2)
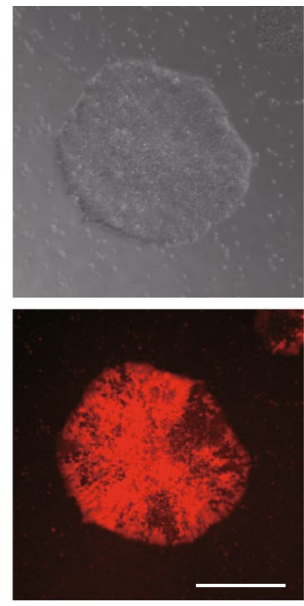

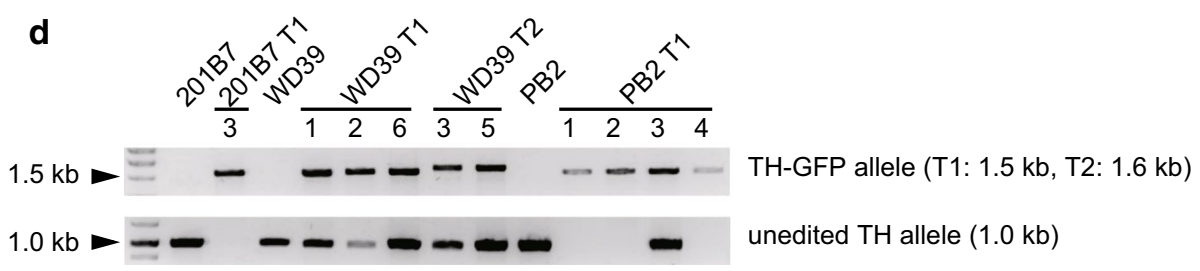

e

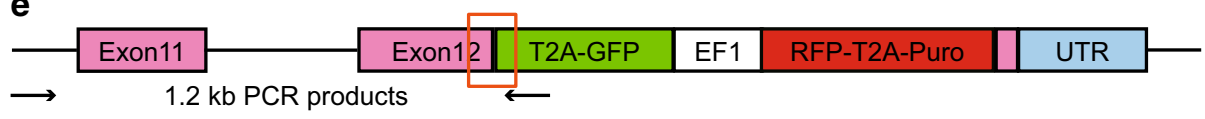
- Adenine - Thymine - Guanine - Cytosine

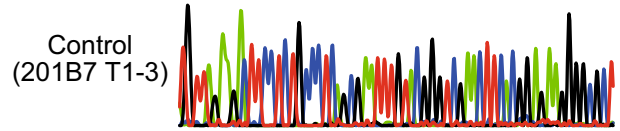

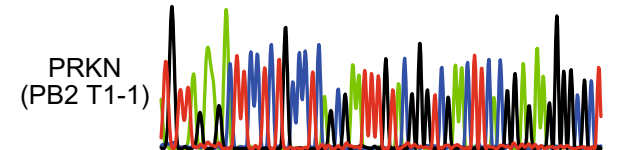
Control
WD39 T1-2)

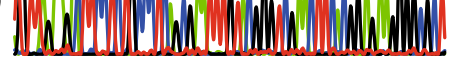

(PB2 T1-4) 
Accutase (Nacalai Tesque), and 15,000 cells were analyzed using Guava easyCyte Flow Cytometer (Luminex).

To examine the mitochondrial membrane potential and active mitochondrial mass for flow cytometer, differentiated cells were incubated for $30 \mathrm{~min}$ with $5 \mathrm{nM}$ TMRM (Thermo Fisher Scientific) and 10 nM Mitotracker Deep Red (Thermo Fisher Scientific). The cells were detached from culture plates with Accutase (Nacalai Tesque), and 3000 cells were analyzed using FACS Aria IIIu (BD Biosciences).

\section{Correlative light-electron microscopy}

For CLEM analysis, dissociated neurospheres were reseeded on gridded glass-bottom $\mu$-dishes (ibidi) or gridded coverslips (Matsunami) after coating them with poly-L-ornithine and fibronectin. On day 7-10 of culture, cells were fixed in $2 \%$ paraformaldehyde, $0.5 \%$ glutaraldehyde, and $50 \mathrm{mM}$ sucrose in $0.1 \mathrm{M}$ phosphate buffer. Brightfield and fluorescent images were then taken using a BZ-X710 fluorescence microscope (Keyence). Next, cells were fixed in $2 \%$ glutaraldehyde and $50 \mathrm{mM}$ sucrose in $0.1 \mathrm{M}$ phosphate buffer, followed by post fixation with $1 \%$ osmium tetroxide. Fixed cells were dehydrated and embedded in Epon812 (Oken Shoji). Ultrathin sections were cut with an ultramicrotome UC6 (Leica) and mounted on glass coverslips. Sections were stained with uranyl acetate and lead citrate, and these coverslips were then coated with carbon using a carbon coater CADE-4T (Meiwa Fosis). Specimens were examined with a scanning electron microscope Helios NanoLab 660 (FEI).

\section{Experimental design and statistical analysis}

The quantitation of fluorescent images was performed for 10 images per iPSC line. The quantitation of electron micrographs was performed in 9-15 cells per iPSC line. GraphPad Prism 8 software was used for statistical analysis. Differences among the groups were evaluated using the unpaired $t$-test or two-way ANOVA with Šidák's multiple comparisons test, as appropriate. A P-value of less than 0.05 was considered significant.

\section{Results}

\section{Generation of control and PRKN-mutated patient TH reporter iPSC lines}

To generate $\mathrm{TH}$ reporter iPSC lines via the CRISPR/Cas9 system, we designed sgRNA expression vectors. First, we determined a target site with less off-target (Target 1) and a target site near the stop codon (Target 2) for Cas9 nuclease among the exon 12 sequence of the $T H$ gene (Fig. 1a). The sgRNA expression vectors were designed by inserting the PCR products, including the Target 1 or 2 sequence, into an $\mathrm{RiH}$ vector [29]. Next, we designed the donor vector for insertion into the target sites by
Table 1 Screening of control and PRKN-mutated TH-GFP iPSC lines by PCR analysis for TH-GFP allele

\begin{tabular}{llll}
\hline Original iPSC line & Target site & $\begin{array}{l}\text { No. of RFP- } \\
\text { positive colonies }\end{array}$ & $\begin{array}{l}\text { No. of colonies } \\
\text { with TH-GFP } \\
\text { allele }\end{array}$ \\
\hline Control (201B7) & Target 1 & 6 & 1 \\
& Target 2 & 5 & 0 \\
Control (WD39) & Target 1 & 3 & 3 \\
& Target 2 & 6 & 2 \\
PRKN (PB2) & Target 1 & 6 & 4 \\
& Target 2 & 9 & 0 \\
\hline
\end{tabular}

homologous recombination (Fig. 1b). To avoid a severe impact on the function of $\mathrm{TH}$ by the reporter gene, we chose an HR130PA-1 vector, which is a T2A-based bicistronic expression vector. The $5^{\prime}$ or $3^{\prime}$ homology arms flanking the cleavage site were respectively inserted into each end of the HR130PA-1 vector (T2A-GFP-pA-loxPEF1 $\alpha$-RFP-T2A-Puro-pA-LoxP-MCS).

The sgRNA, Cas9, and donor vectors were then introduced into the control lines (201B7, WD39) and the PD patient line (PB2). After 8-9 days of puromycin selection, several RFP-positive colonies were observed among the puromycin-resistant colonies in each line, suggesting that the donor sequence was introduced into any site of genomic DNA in the iPS colonies (Fig. 1c). We picked up the RFP-positive colonies and divided each colony into two: one part for DNA isolation and one part for expanding. Purified DNA was amplified using PCR primers for the detection of the TH-GFP allele (Additional file 1: Table S1), to screen the clones that had the GFP sequence at the target site in the $T H$ gene. PCR analysis showed that 8 of the 15 RFP-positive colonies that were transfected with the Target 1 sgRNA vector had the GFP sequence at the target site in the $T H$ gene (Table 1 ). In contrast, only 2 of the 20 RFP-positive colonies that were transfected with the Target 2 sgRNA vector had the GFP sequence at the target site in the $T H$ gene (Table 1). The low efficiency of the Target 2 sgRNA is likely caused by its high number of off-targets relative to Target 1 . To determine whether the clones were homozygous or heterozygous for the TH-GFP allele, clones with the TH-GFP allele were expanded and further analyzed for unedited TH allele using PCR. As a result, a B7 knock-in clone (201B7 T1-3) and three of the four PB2 knock-in clones (PB2 T1-1, -2, and -4) carried homozygous TH-GFP alleles (Fig. 1d). In contrast, all of the WD39 knock-in clones carried heterozygous TH-GFP alleles (Fig. 1d). Sanger sequencing demonstrated that the T2A-GFP sequence was correctly inserted at the cleavage site in all of four knock-in iPSC lines used in this study: a control 
(See figure on next page.)

Fig. 2 Characterization of dopaminergic neurons differentiated from TH-GFP iPSC lines. a The brightfield and fluorescent images at day 7 after reseeding the neurospheres demonstrated GFP expression in some of the differentiated neurons derived from TH-GFP iPSC lines. "PRKN" represents PRKN-mutated patient. Scale bar, $50 \mu \mathrm{m}$. b Flow cytometric analysis showed GFP-positive fractions in the TH-GFP iPSC-derived neurons against the original iPSC-derived neurons at day 9 after reseeding the neurospheres. "PRKN" represents PRKN-mutated patient. c Immunofluorescence staining for GFP and TH confirmed similar expression patterns among TH-GFP iPSC-derived neurons. "PRKN" represents PRKN-mutated patient. Scale bar, $20 \mu \mathrm{m}$. $\mathbf{d}$ Pie graphs showing quantitative analysis of the proportion of TH+GFP+ (yellow areas), TH-GFP+ (green areas), TH+GFP- or THGFP - (white areas) cells in the total DAPI+ cells from the immunofluorescence images ( $n=10$ per iPSC line) in (c). There were no TH+GFP- cells derived from any TH-GFP iPSC lines. "PRKN" represents PRKN-mutated patient. Values are shown as the mean \pm SEM

homozygous line (B7 T1-3), a control heterozygous line (WD39 T1-2), and two patient homozygous lines (PB2 T1-1 and -4) (Fig. 1e). Thus, these data demonstrate the successful generation of control and PRKN-mutated patient TH reporter iPSC (TH-GFP iPSC) lines.

\section{Differentiation of the TH-GFP iPSC lines into dopaminergic neurons}

To confirm the expression of the TH-GFP gene, we differentiated the generated TH-GFP iPSC lines into dopaminergic neurons using previously reported the direct neurosphere converting method [14, 33]. On day 7 after reseeding the neurospheres, GFP expression was detected in differentiated neurons derived from all of four TH-GFP iPSC lines (Fig. 2a). Flow cytometric analysis demonstrated that $11.3-42.4 \%$ GFP-positive fractions existed in TH-GFP iPSC-derived neurons on day 9 after reseeding the neurospheres (Fig. 2b). In the WD39 T1-2 neurons, which carried heterozygous TH-GFP alleles, GFP expression levels were comparable to those of other homozygous reporter lines (Fig. 2b).

To examine the specificity of the TH reporter, we stained the TH-GFP iPSC-derived neurons with anti$\mathrm{TH}$ and anti-GFP antibodies and confirmed a strong colocalization of TH and GFP (Fig. 2c). We measured the proportion of TH/GFP double-positive, TH or GFP single-positive, and TH/GFP double-negative cells in the immunofluorescence images. TH-positive/GFP-negative and TH-negative/GFP-positive cells were hardly detected in the immunofluorescence images, suggesting that the generated TH-GFP iPSC lines had high specificity for the TH reporter (Fig. 2c, d). The proportion (39.9-46.8\%) of TH-positive dopaminergic neurons in the total differentiated cells was comparable to that of the original iPSC lines [13] (Fig. 2d). However, the proportions of GFP-positive neurons were lower when analyzed in flowcytometry (11.3-42.4\%) (Fig. 2b) compared with the quantitation of TH/GFP-positive dopaminergic neurons in immunostaining (39.9-46.8\%) (Fig. 2d). This discrepancy probably resulted from the higher detection of $\mathrm{TH} /$ GFP by immunostaining in neurons expressing low levels of TH/GFP.
To characterize the TH/GFP-double negative cells, we stained the TH-GFP iPSC-derived cells with anti- $\beta 3$ tubulin and anti-Nestin antibodies. Both $\beta 3$-tubulinpositive/TH-negative cells and Nestin-positive/ TH-negative cells existed in the all lines of TH-GFP iPSC-derived neurons (Additional file 2: Figure S1). This result suggests that the TH/GFP-negative cell population contains other neuronal subtypes and neural progenitor cells (Additional file 2: Figure S1, Fig. 3a). Together, these findings indicate that our generated control and PRKN-mutated patient TH-GFP iPSC lines can be used for dopaminergic neuron-specific analyses of control and PRKN-mutated patient.

\section{CLEM analysis of mitochondrial morphology in GFP-positive and/or GFP-negative cells derived from TH-GFP iPSCs}

The death of dopaminergic neurons in PRKN-related PD is assumed to be caused by the accumulation of damaged mitochondria $[4,5]$. To investigate the mitochondrial morphology specific to dopaminergic neurons under normal conditions, we planned to compare the ultrastructure of dopaminergic neurons with non-dopaminergic neurons. For this purpose, we used a CLEM strategy, which allows to gain not only the location of dopaminergic and non-dopaminergic neurons but also the fine ultrastructural information, because the strong fixation for electron microscopy is performed after fluorescent images are taken (Fig. 3b). The TH-GFP iPSC-derived neurons were seeded on gridded coverslips, and brightfield and fluorescent images were taken before the cells were fixed strongly and processed for electron microscopy (Fig. 3b). For the CLEM analyses, we selected cells with either strong GFP expression or no GFP expression at all (Fig. 3a), because in the fluorescent imaging, neurons with weak expression of TH /GFP were difficult to detect. We marked 10-15 GFP-positive and -negative cells per TH-GFP cell line on the brightfield and fluorescent images (Fig. 3c). We then searched for the marked cells on the ultrathin sections, with help from grid patterns and cell shapes, under a scanning electron microscope (Fig. 3d). 

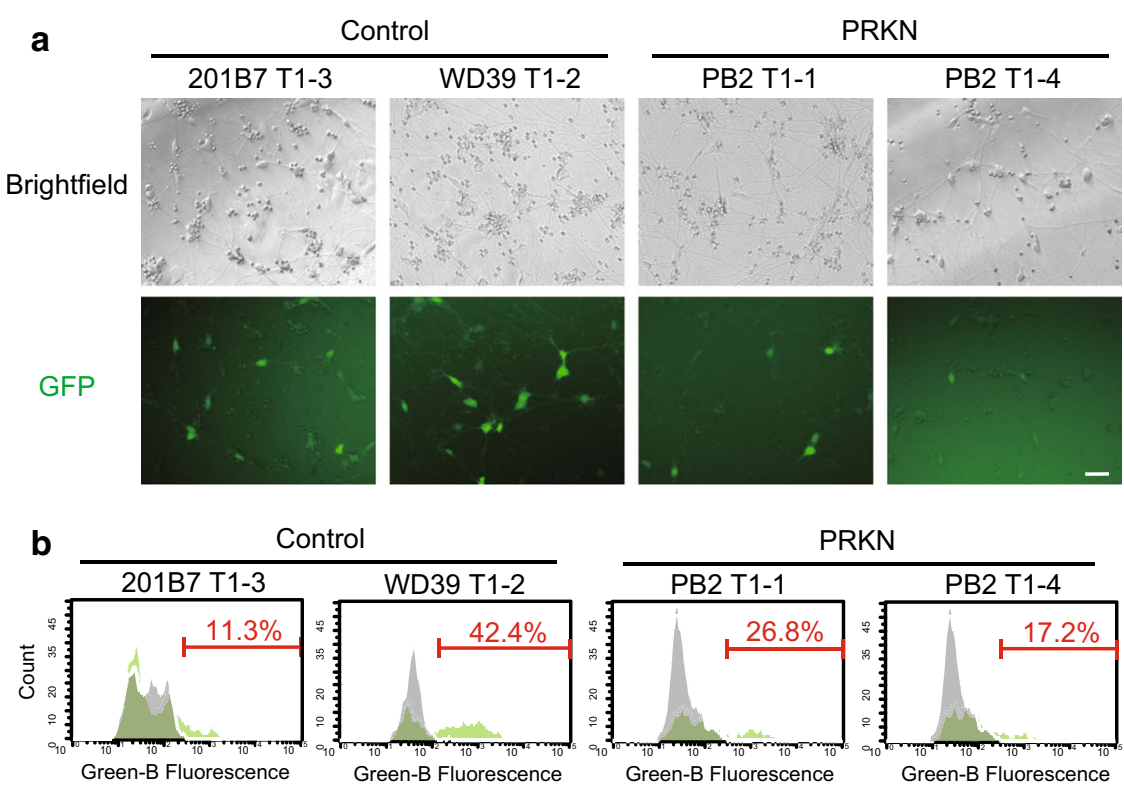

c

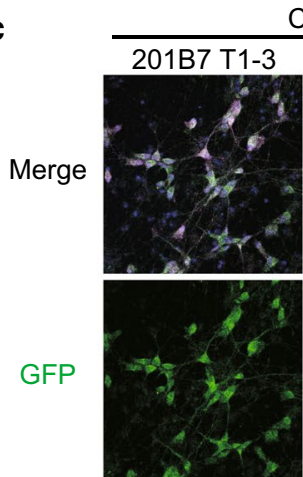

Control
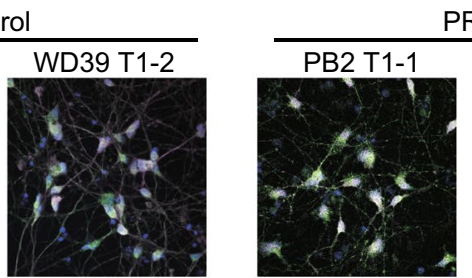

PRKN
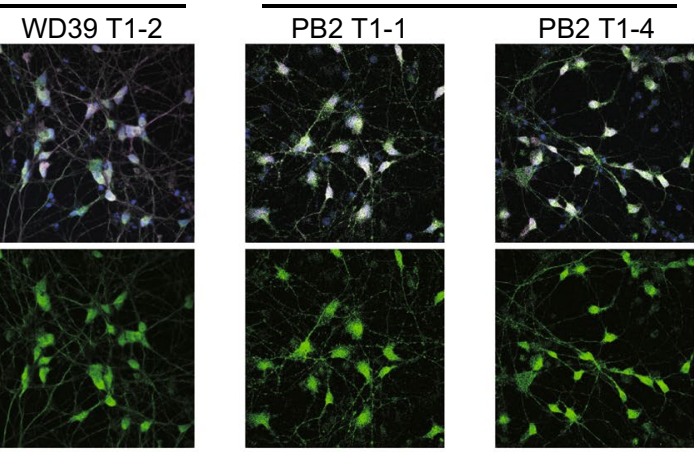

TH
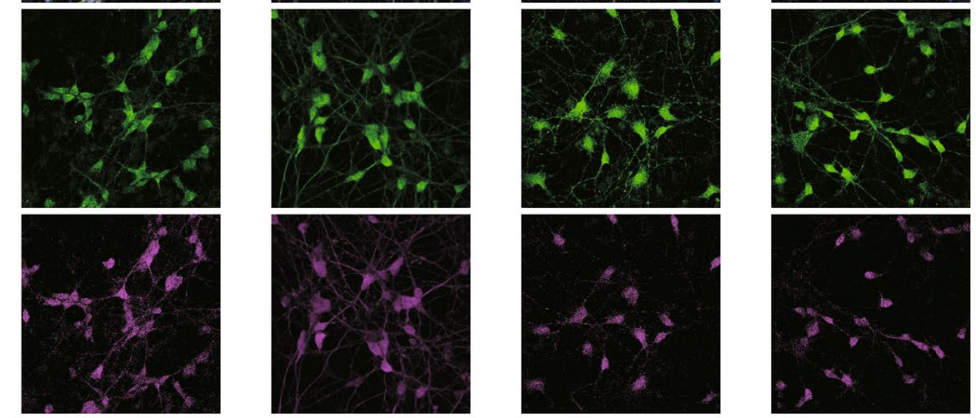

DAPI
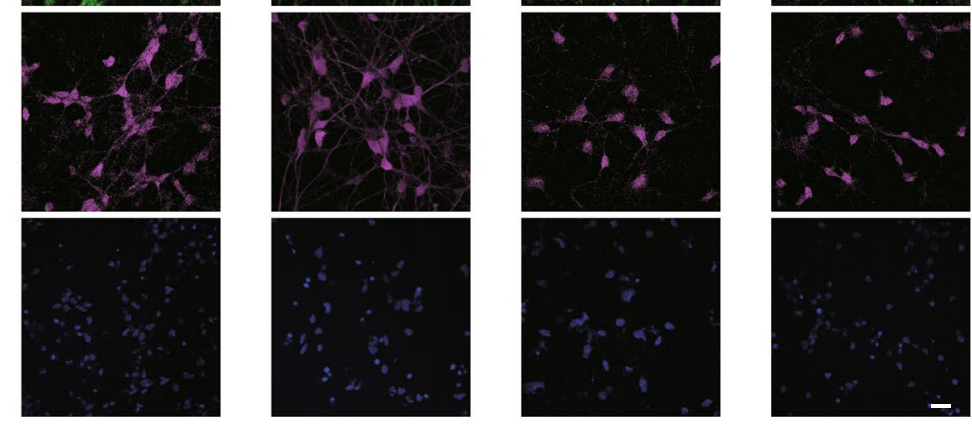

d
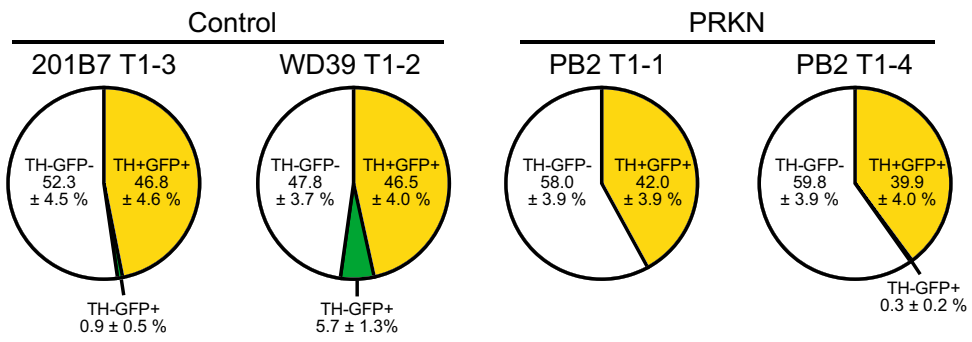
(See figure on next page.)

Fig. 3 CLEM analysis of GFP-positive and GFP-negative cells derived from TH-GFP iPSCs. a Diagram representing the comparative analysis of GFP-positive and -negative cells in CLEM analysis (Figs. 4 and 7) and live cell imaging (Fig. 5, Additional file 3: Figure S2). Neurons expressing high levels of GFP (green in the diagram) were defined as "GFP-positive" cells. Cells expressing no levels of GFP and with a neuronal shape, which were probably other neuronal subtypes or neural progenitors (white in the diagram), were defined as "GFP-negative" cells. Neurons expressing low levels of GFP (light green in the diagram) were not used in either CLEM analysis or live cell imaging. b Scheme of the CLEM procedure. Neurospheres differentiated from TH-GFP iPSCs were dissociated and reseeded on the gridded coverslips. After 7-10 days of culture, the differentiated neurons consisted of a mixture of GFP-positive (green) dopaminergic neurons and GFP-negative (white) non-dopaminergic neurons. Brightfield and fluorescent images were taken of cells on the gridded coverslips, and the cells were further fixed and flat-embedded for electron microscopic analysis. The cells of interest in the ultrathin sections were identified with help from the grid patterns and cell shapes using an SEM. c The GFP (right) and merged (brightfield + GFP, left) images of TH-GFP iPSC-derived cells on the gridded coverslips. The white box indicates the origin of the enlarged image (c, left). Scale bar, $100 \mu \mathrm{m}$. d The brightfield and GFP (left), GFP and SEM (center), and SEM (right) images. Asterisks and arrows indicate GFP-positive and -negative cells, respectively. Scale bar, 20 mm

Almost all of the marked cells were found and observed for mitochondrial morphology in the ultrathin sections. Under normal conditions mitochondrial structural changes were not found in GFP-positive and -negative cells derived from control and PRKN-mutated iPSC lines, as far as we observed using CLEM analysis. On the other hand, CLEM analysis revealed that mitochondrial size tended to be smaller in the GFP-positive dopaminergic neurons compared with the GFP-negative non-dopaminergic neurons, in all of the TH-GFP lines (Fig. 4a, b). To confirm this finding, we measured the area and major axis of each mitochondrion in the electron microscope images. Quantitation of the mitochondrial area showed that mitochondria in GFP-positive dopaminergic neurons were significantly smaller than in GFP-negative non-dopaminergic neurons in both the control and PRKN-mutated lines (Fig. 4c control, $P<0.0001$; Fig. 4c PRKN, $P=0.0064)$. The mitochondrial major axes in GFP-positive dopaminergic neurons were also significantly shorter than in GFP-negative non-dopaminergic neurons in control lines (Fig. $4 \mathrm{~d}$ control, $P<0.0001$ ). In $P R K N$-mutated lines, the mitochondrial major axis in GFP-positive dopaminergic neurons tended to be shorter than that in GFP-negative non-dopaminergic neurons, but this difference was not statistically significant (Fig. 4d PRKN, $P=0.1651)$. There were significant differences of mitochondrial area and major axis in non-dopaminergic neurons between control and $P R K N$-mutated lines (Fig. 4c, $P<0.0001$; Fig. 4 d, $P<0.0001$ ), suggesting that the loss of Parkin altered the mitochondrial morphology in non-dopaminergic neurons. Together, these results indicate that the iPSC-derived dopaminergic neurons had smaller mitochondria than the non-dopaminergic neurons in both the control and PRKN-mutated lines.

\section{Mitochondrial membrane potential in GFP-positive and -negative cells derived from TH-GFP iPSCs}

To further investigate whether mitochondrial function in dopaminergic neurons is lower than in non-dopaminergic neurons, we compared mitochondrial membrane potentials in living GFP-positive dopaminergic neurons and GFP-negative non-dopaminergic neurons using TMRM, a fluorescent probe for monitoring the mitochondrial membrane potential. Live cell imaging showed that the fluorescent signal of TMRM in GFP-positive dopaminergic neurons was remarkably lower than in GFP-negative non-dopaminergic neurons in both the control and PRKN-mutated lines (Fig. 5a). Quantitation of the mean fluorescent intensity (MFI) of TMRM confirmed that the mitochondrial membrane potential per cell in dopaminergic neurons was significantly lower than in non-dopaminergic neurons (Fig. $5 \mathrm{~b}$ left, $P<0.0001$; Fig. 5c left, $P=0.0015$ ).

Next, we quantified the MFI of Mitotracker Deep Red, which is a fluorescent dye of mitochondria. We then normalized the MFI of TMRM to the MFI of Mitotracker Deep Red to examine the membrane potential per mitochondria (Fig. $5 \mathrm{~b}$ center, $5 \mathrm{~b}$ right, $5 \mathrm{c}$ center, and $5 \mathrm{c}$ right). The mitochondrial membrane potential normalized to the mitochondrial mass was also significantly lower in dopaminergic neurons than in non-dopaminergic neurons in the control lines (Fig. 5b right, $P=0.0023$ ). In the PRKN-mutated lines, the mitochondrial membrane potential normalized to the mitochondrial mass tended to be lower in dopaminergic neurons than in non-dopaminergic neurons, but this difference was not significant (Fig. 5 c right, $P=0.0551$ ).

To further confirm the mitochondrial membrane potentials in dopaminergic and non-dopaminergic neurons, we stained the differentiated cells with TMRM and Mitotracker Deep Red for flow cytometric analysis. Flow cytometric analysis showed that $6.5 \%-28.6 \%$ GFP-positive fractions existed in the differentiated cells derived from each TH-GFP iPSC lines (Additional file 3: Figure S2a). Consistent with the results from live cell imaging, TMRM-GFP cytograms showed that GFP-positive populations had the lower TMRM signal compared to GFP-negative populations in the control lines (Additional 


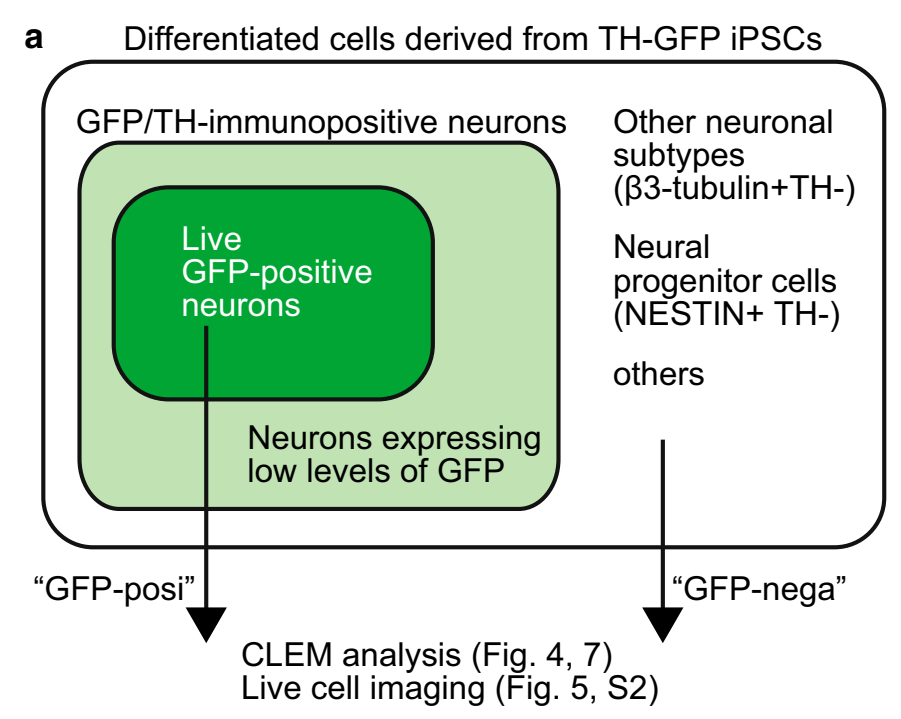

b

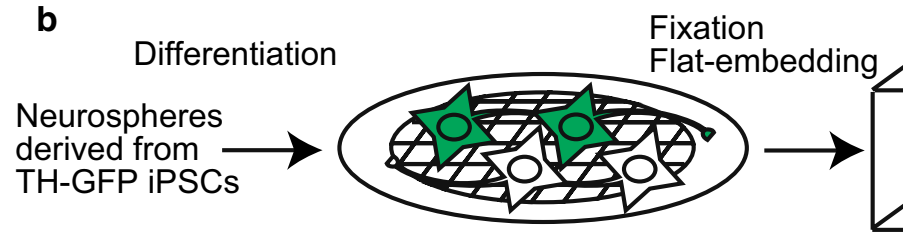

Reseeding on a gridded coverslips and taking a fluorescent image 7-10 days after reseeding (Fig. 3b, 3c left)

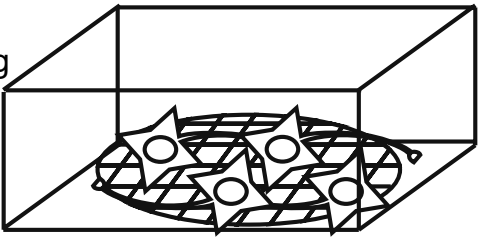

Cutting and observing ultrathin sections under SEM (Fig. 3c right)

c
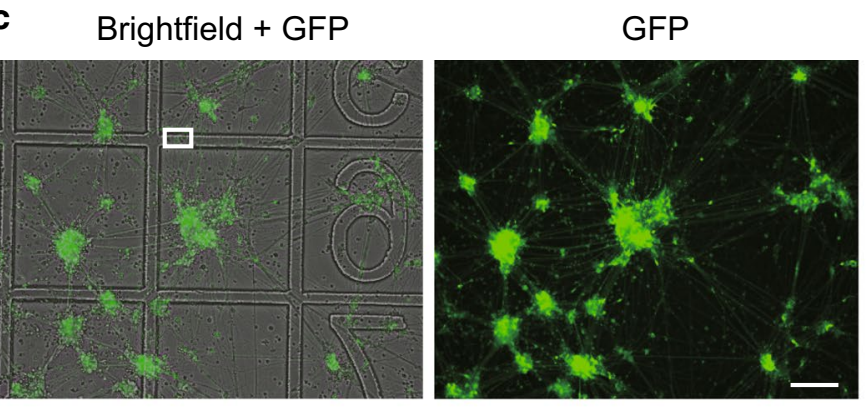

d Brightfield + GFP

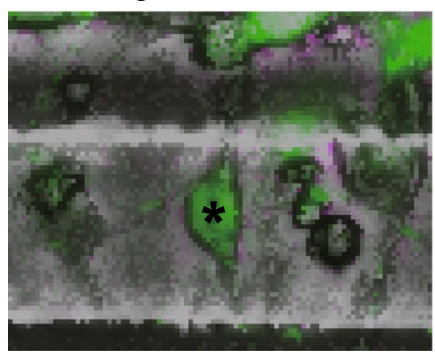

$\mathrm{GFP}+\mathrm{SEM}$

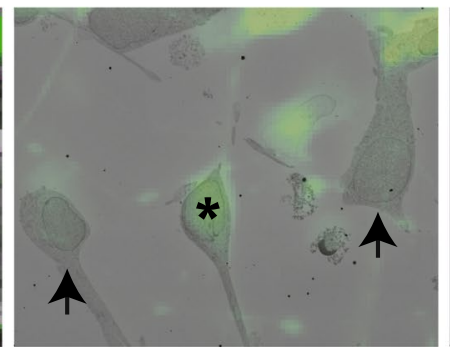

SEM

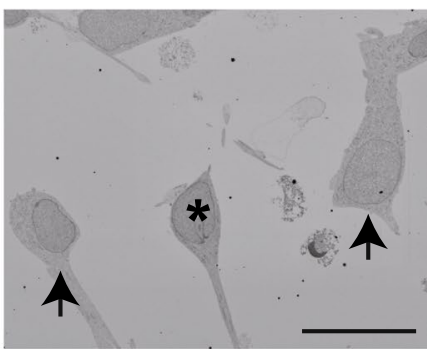



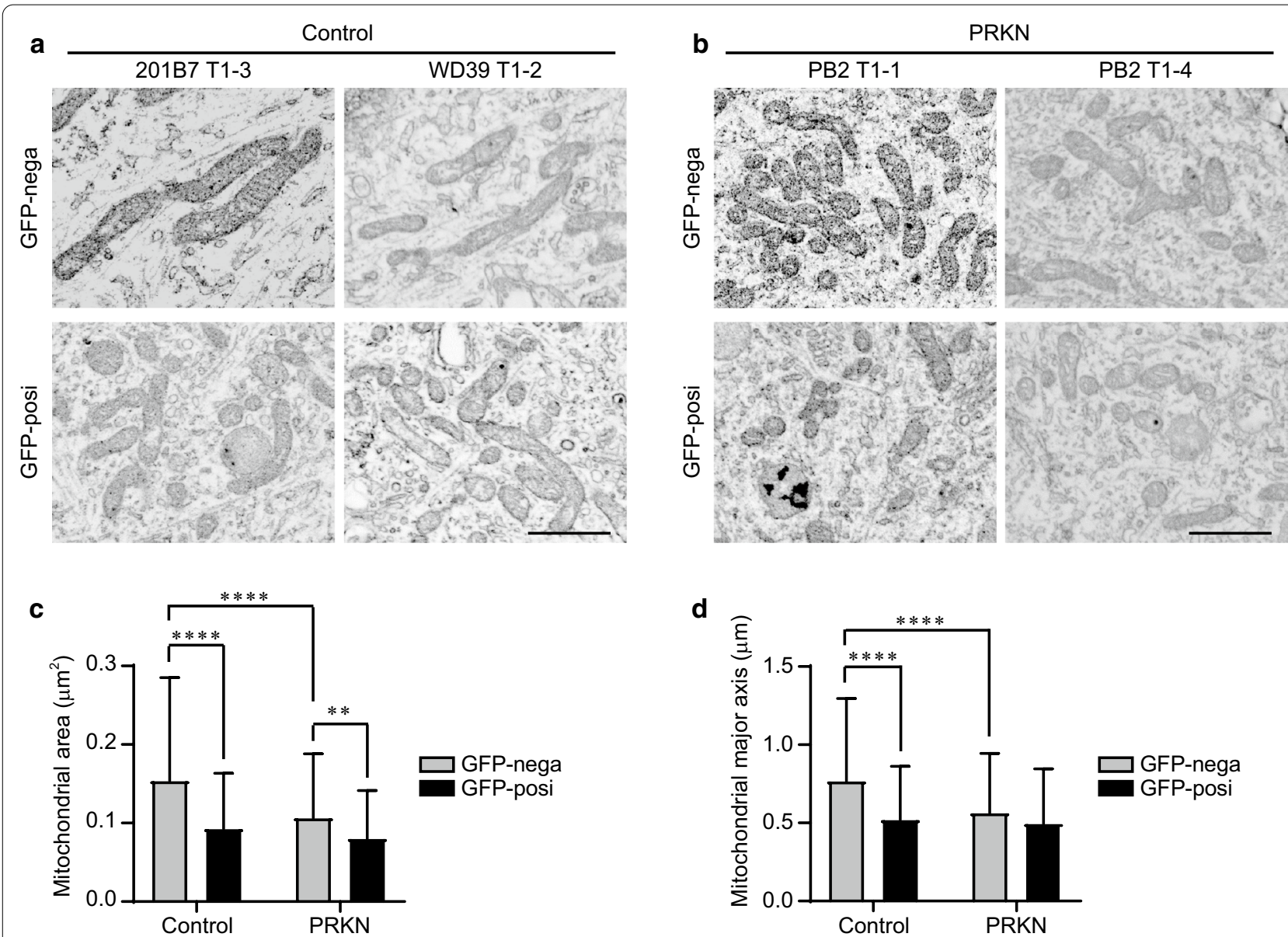

Fig. 4 Cell type-specific mitochondrial morphology in dopaminergic neurons derived from TH-GFP iPSCs. a Representative SEM images of GFP-negative non-dopaminergic neurons (top) and GFP-positive dopaminergic neurons (bottom) derived from control TH-GFP iPSCs. Scale bar, $1 \mu \mathrm{m}$. b Representative SEM images of GFP-negative non-dopaminergic neurons (top) and GFP-positive dopaminergic neurons (bottom) derived from PRKN-mutated TH-GFP iPSCs. "PRKN" represents PRKN-mutated patient. Scale bar, 1 m. c Quantitative analysis of the mitochondrial area from the SEM images of GFP-negative and -positive cells derived from control and PRKN-mutated TH-GFP iPSCs. "PRKN" represents PRKN-mutated patient. Mitochondrial area (control GFP-nega; $n=199$, control GFP-posi; $n=198$, PRKN GFP-nega; $n=215$, PRKN GFP-posi; $n=200$ ) was measured using ImageJ. Values are shown as the mean \pm SD. Statistical significance was evaluated using the two-way ANOVA with Šidák's multiple comparisons test. ${ }^{* *} P<0.01,{ }^{* * *} P<0.0001$. There was no significant difference in the mitochondrial area between control and $P R K N$-mutated GFP-positive cells $(P=0.298)$. $\mathbf{d}$ Quantitative analysis of the mitochondrial major axis from the SEM images of GFP-negative and -positive cells derived from control and PRKN-mutated TH-GFP iPSCS. "PRKN" represents PRKN-mutated patient. The mitochondrial major axis (control GFP-nega; $\mathrm{n}=199$, control GFP-posi; $n=198$, PRKN GFP-nega; $n=215$, PRKN GFP-posi; $n=200$ ) was measured using ImageJ. Values are shown as the mean \pm SD. Statistical significance was evaluated using the two-way ANOVA with Šidák's multiple comparisons test. ${ }^{* * *} P<0.0001$. There was no significant difference in the mitochondrial major axis between the GFP-positive and -negative PRKN-mutated cells $(P=0.1651)$. There was no significant difference in the mitochondrial major axis between control and PRKN-mutated GFP-positive cells $(P=0.7795)$

file 3: Figure S2b). Quantitation of the MFI of TMRM demonstrated that the mitochondrial membrane potentials per cell/mitochondria in GFP-positive dopaminergic neurons were significantly lower than that in GFP-negative non-dopaminergic neurons in the control lines (Additional file 3: Figure S2c control left, $P=0.0169$; Figure S2c control right, $P=0.0315)$. In the $P R K N$-mutated lines, the mitochondrial membrane potentials per cell/ mitochondria in GFP-positive dopaminergic neurons tended to be lower than that in GFP-negative non-dopaminergic neurons, although these differences were not significant (Additional file 3: Figure S2c PRKN left, $P=0.5567$; Figure S2c PRKN right, $P=0.1382$ ). Contrary to the results from live cell imaging, the mitochondrial mass in $P R K N$-mutated dopaminergic neurons was significantly higher than that in $P R K N$-mutated non-dopaminergic neurons (Additional file 3: Figure S2c PRKN center, $P=0.0438$ ). 


\begin{abstract}
(See figure on next page.)
Fig. 5 Live cell imaging of mitochondrial membrane potential in dopaminergic neurons derived from TH-GFP iPSCs. a Live cell imaging of dopaminergic and non-dopaminergic neurons derived from control and PRKN-mutated TH-GFP iPSCs. Arrowheads indicate weak TMRM signals in GFP-positive dopaminergic neurons. Arrows indicate strong TMRM signals in GFP-negative non-dopaminergic neurons. "PRKN" represents PRKN-mutated patient. Scale bar, 5 um. b Quantitative analysis of the MFI of TMRM (left), Mitotracker DeepRed (center), and TMRM normalized to Mitotracker DeepRed (right) in control GFP-positive cells relative to control GFP-negative cells in fluorescence images ( $n=20$ images). The MFI was measured using the surrounding cytoplasmic area in ZEN software. Values are shown as the mean \pm SEM. Statistical significance was evaluated using the unpaired two-tailed $t$-test. ${ }^{* *} P<0.01,{ }^{* * * *} P<0.0001$. c Quantitative analysis of the MFI of TMRM (left), Mitotracker DeepRed (center), and TMRM normalized with Mitotracker DeepRed (right) in PRKN-mutated GFP-positive cells relative to PRKN-mutated GFP-negative cells in fluorescence images ( $n=20$ images). "PRKN" represents PRKN-mutated patient. Values are shown as the mean \pm SEM. Statistical significance was evaluated using the unpaired two-tailed $t$-test. ${ }^{*} P<0.01$. There were no significant differences in the MFI of Mitotracker and TMRM/Mitotracker between GFP-positive and -negative cells $\left(b^{\prime \prime} ; P=0.1163, b^{\prime \prime \prime} ; P=0.0551\right)$
\end{abstract}

These results indicate that iPSC-derived dopaminergic neurons have lower mitochondrial membrane potentials not only at the individual mitochondrial level but also at the cellular level. Nevertheless, the differences in mitochondrial function and size between dopaminergic and non-dopaminergic neurons in the control lines were more significant than in the PRKN-mutated lines. This result suggests that the smaller size and lower function of mitochondria in the dopaminergic neurons are probably not the result of the PRKN mutation, but are rather characteristic of dopaminergic neurons themselves.

\section{Cell death of dopaminergic neurons derived}

from $P R K N$-mutated patient TH-GFP iPSC lines under CCCP A previous study has reported that PRKN-mutated dopaminergic neurons show increased cell death compared with control lines under CCCP treatment for $24 \mathrm{~h}$ [13]. To evaluate the cell death of dopaminergic neurons in the generated control and PRKN-mutated TH-GFP iPSC lines, the TH-GFP iPSC-derived cells were treated with $\mathrm{CCCP}$ for $24 \mathrm{~h}$ and then stained with the antibody against cleaved caspase-3, which is a marker of apoptosis. There were significantly increased numbers of cleaved caspase- 3 -positive cells in the PRKN-mutated dopaminergic neurons compared with both the control dopaminergic neurons (Fig. 6a, b; $P=0.0011$ ) and the $P R K N$-mutated non-dopaminergic neurons (Fig. 6a, b; $P<0.0001$ ). These results suggest that the TH-GFP $P R K N$-mutated patient iPSC line replicates the typical pathological phenotype of PD with PRKN mutations: the preferential loss of dopaminergic neurons.

\section{Electron microscopic analysis of dopaminergic neurons derived from PRKN-mutated TH-GFP iPSCs under CCCP treatment}

As shown above, we were unable to identify any differences in mitochondria between the PRKN-mutated and control lines under normal conditions using CLEM and live cell imaging. CLEM analysis demonstrated that normal condition induced no mitochondrial structural change in both control and PRKN-mutated lines (Fig. 7a). We, therefore, treated the TH-GFP iPSC-derived cells with CCCP for $3.5 \mathrm{~h}$ to detect the initial changes in mitochondrial structure before cell death.

We first confirmed that CCCP treatment for $3.5 \mathrm{~h}$ reduced the mitochondrial membrane potential in control TH-GFP iPSC-derived cells using live cell imaging with TMRM (Additional file 4: Figure S3). Next, we observed mitochondrial ultrastructure in the CCCPtreated dopaminergic neurons using CLEM. CLEM analysis revealed that CCCP treatment in control GFPpositive dopaminergic neurons induced the transformation of mitochondria into ring-shaped (donut-shaped) structures (Fig. 7b control). The mitochondrial morphological changes from tubular forms to ring-shaped forms have previously reported in MEF and HeLa cells under CCCP treatment, and the mitochondria with the characteristic ring-shaped structures are called spherical mitochondria [17, 18, 34, 35]. In contrast, few spherical mitochondria were observed in PRKN-mutated GFP-positive dopaminergic neurons (Fig. 7b PRKN). By calculating the ratio of spherical mitochondria, we demonstrated that the formation of spheroid-shaped mitochondria was significantly reduced in $P R K N$-mutated dopaminergic neurons (Fig. 7c; $P=0.0003$ ). There was no significant difference in the ratio of spherical mitochondria between control and PRKN-mutated lines in nondopaminergic neurons (Fig. 7c; $P=0.1038$ ). The finding indicates that CCCP treatment induces significant differences in mitochondrial structure between control and PRKN-mutated dopaminergic neurons.

\section{Discussion}

In the present study, we first established TH-GFP iPSC lines from not only controls but also a patient with a PRKN mutation and first analyzed these cells using live cell imaging and CLEM to evaluate the structure and function of mitochondria specifically in dopaminergic neurons. Several studies have reported the establishment of $\mathrm{TH}$ reporter iPSC lines using gene editing technology 

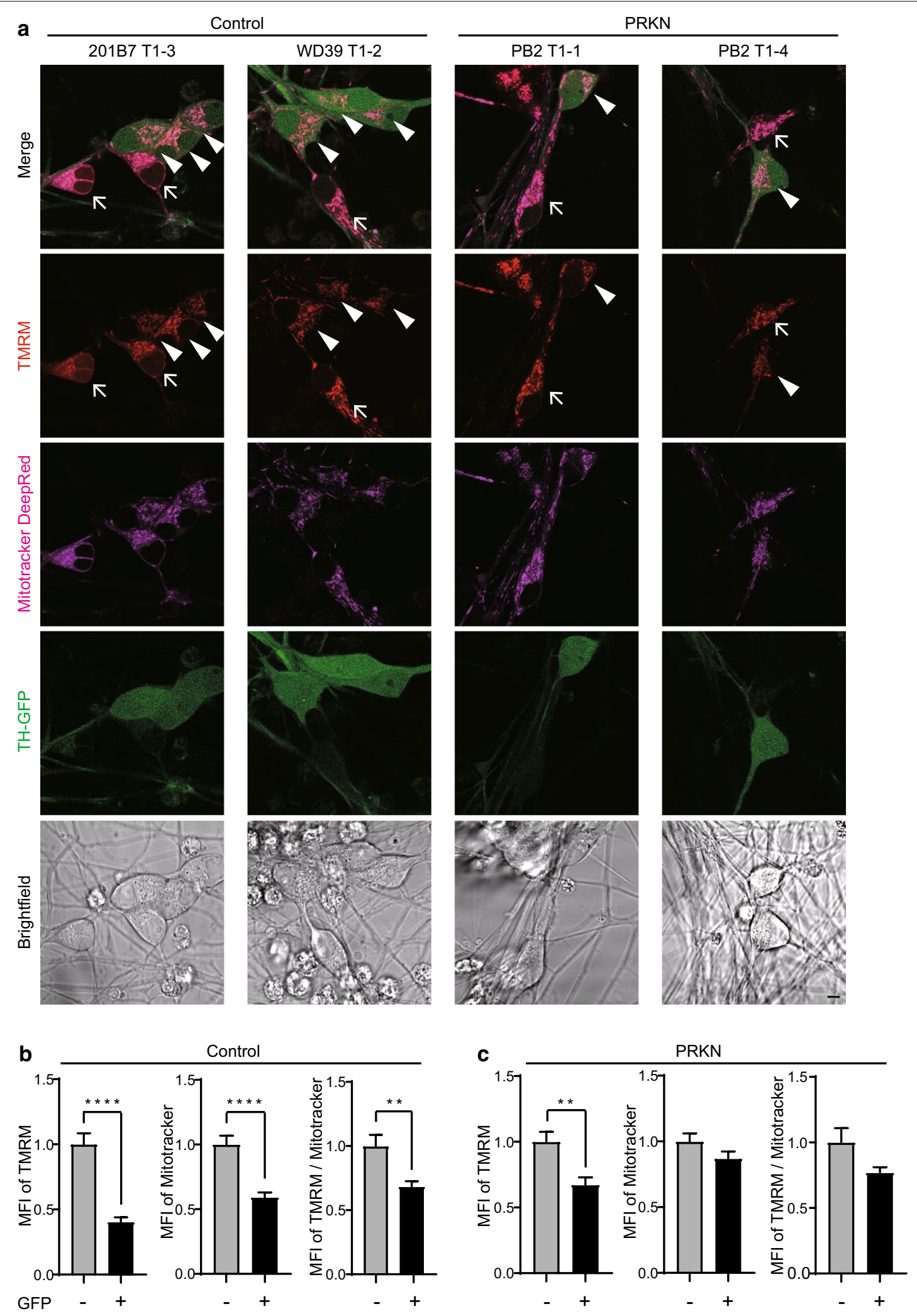


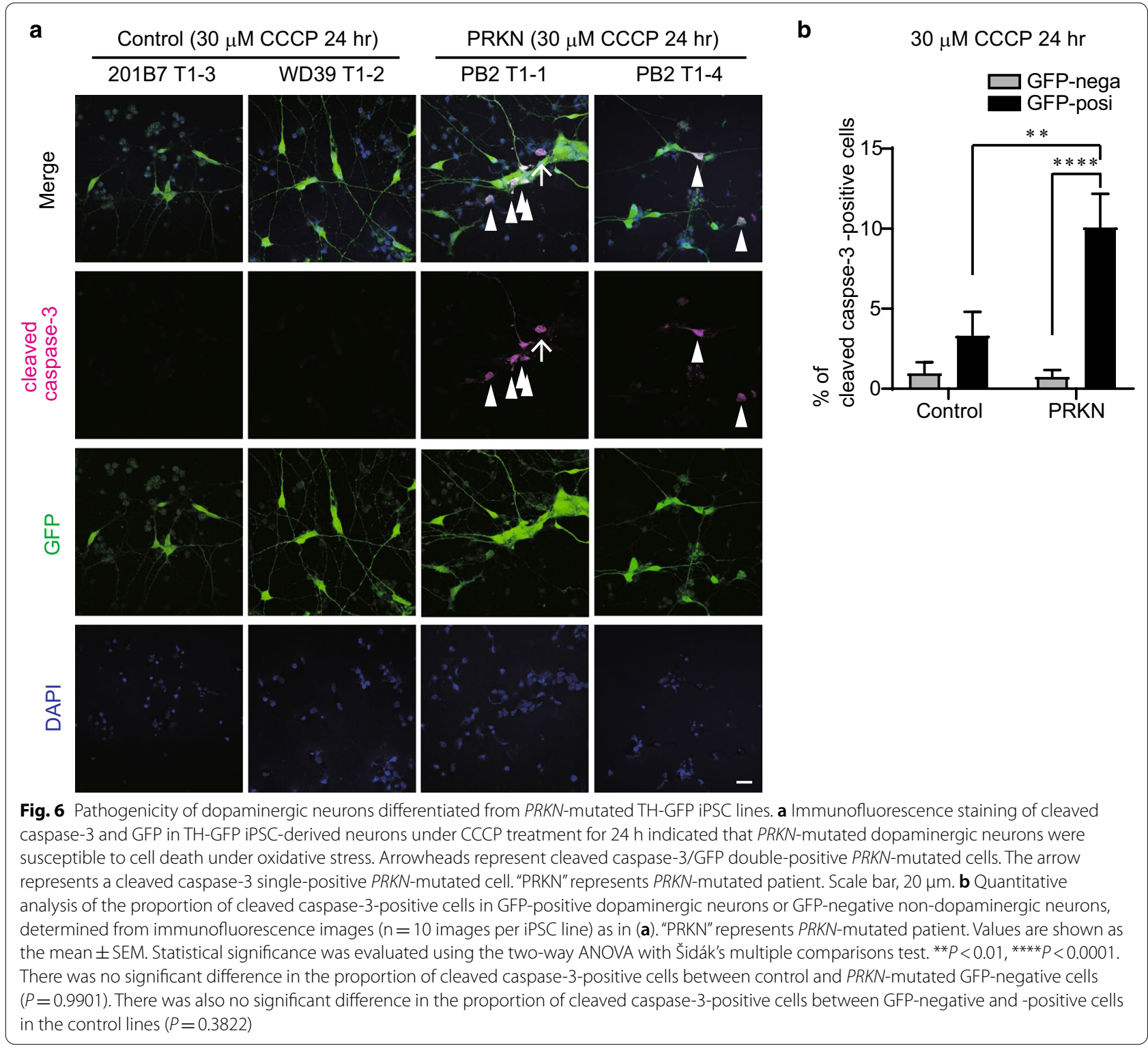

(See figure on next page.)

Fig. 7 Spheroidal formation of mitochondria in iPSC-derived dopaminergic neurons under CCCP treatment. a SEM images of GFP-positive dopaminergic neurons derived from control and PRKN-mutated TH-GFP iPSC lines under normal conditions. "PRKN" represents PRKN-mutated patient. No spherical mitochondria of total mitochondria (control GFP-nega; $n=745$, control GFP-posi; $n=1427$, PRKN GFP-nega; $n=811$, PRKN GFP-posi; $n=1178$ ) was observed in the SEM images of differentiated cells. Scale bar, $1 \mu \mathrm{m}$. b SEM images of GFP-positive dopaminergic neurons derived from control and PRKN-mutated TH-GFP iPSC lines under CCCP treatment for $3.5 \mathrm{~h}$. Mitochondrial structures painted cyan and indicated by arrowheads represent spherical mitochondria. "PRKN" represents PRKN-mutated patient. Scale bar, 1 mm. The inset shows an image of one of the spherical mitochondria without painting. Scale bar of the inset, $500 \mathrm{~nm}$. c Quantitative analysis of the percentage of spherical mitochondria in total mitochondria (control GFP-nega; $n=562$, control GFP-posi; $n=850$, PRKN GFP-nega; $n=736$, PRKN GFP-posi; $n=774$ ) from the SEM images of differentiated cells, as in (a). "PRKN" represents PRKN-mutated patient. Values are shown as the mean \pm SEM. Statistical significance was evaluated using the two-way ANOVA with Šidák's multiple comparisons test. ${ }^{* * *} P<0.001$. There was no significant difference in the percentage of spherical mitochondria between control and PRKN-mutated GFP-negative cells $(P=0.1038)$. There was also no significant difference in the percentage of spherical mitochondria between GFP-negative and GFP-positive cells (control; $P=0.8825, \mathrm{PRKN} ; P=0.2222$ ) 


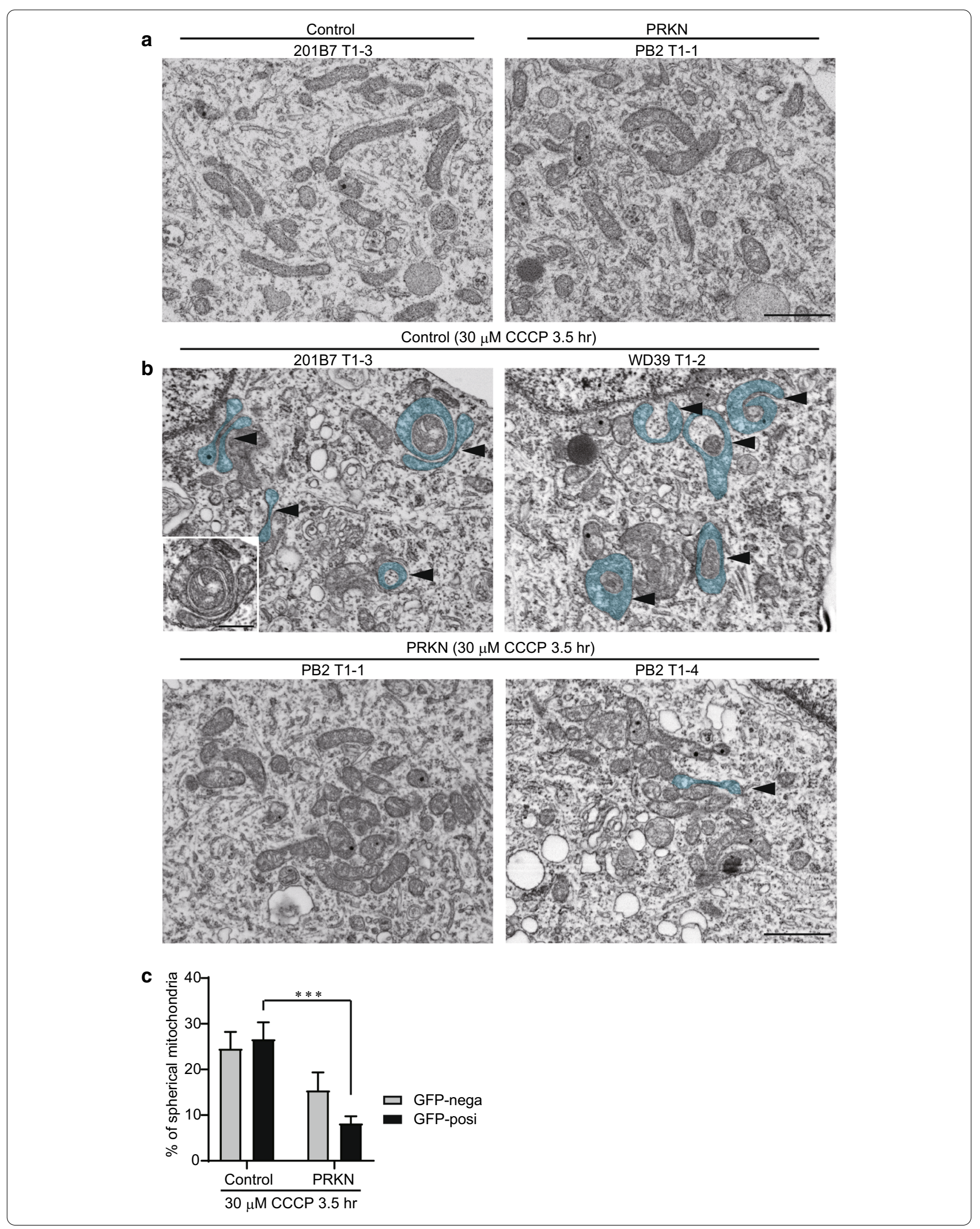


for transcriptome profiling or the electrical properties of dopaminergic neurons [23-25]. Immunohistochemistry confirmed that the specificity of our TH-GFP iPSC lines was sufficient for the identification of dopaminergic neurons, similar to that of other knock-in reporters using genome editing technology. There was a small variation in the proportion of GFP-positive cells, possibly due to the difference in the cell line-specific gene expression pattern. The PRKN-mutated TH-GFP iPSC lines replicated the preferential death of dopaminergic neurons caused by CCCP treatment for $24 \mathrm{~h}$. This result indicates that our established control and PRKN-mutated TH-GFP iPSC lines are useful for analyzing the specific phenotype of dopaminergic neurons in patients with PRKN mutations. We aimed to use the TH-GFP iPSC lines for CLEM analysis of mitochondrial ultrastructure, because mitochondrial ultrastructure has not been clearly examined in control and PRKN-mutated patient dopaminergic neurons in previous studies.

CLEM analysis and live cell imaging under normal conditions demonstrated that dopaminergic neurons had smaller and less functional mitochondria compared with non-dopaminergic neurons in both the control and $P R K N$-mutated lines. Our results regarding mitochondrial size in dopaminergic neurons are consistent with those of a previous study, which reported significantly smaller mitochondria in substantia nigra dopaminergic neurons compared with non-dopaminergic neurons in the mouse brain [36]. Our data present the first in vitro evidence of smaller mitochondria in human iPSC-derived dopaminergic neurons.

Our quantitation of mitochondrial size in CLEM also provided evidence that the mean mitochondrial size in cells derived from the $P R K N$-mutated patient tended to be smaller than that of control cells in both dopaminergic and non-dopaminergic neurons. Similarly, a previous study of iPSC-derived neurons using electron microscopy showed that control neurons have long cylindrical mitochondria compared with PRKN-mutated neurons, although mitochondrial ultrastructure was not specifically analyzed in dopaminergic neurons [12]. Together, these results suggest that mitochondrial size in both dopaminergic and non-dopaminergic neurons may be affected by PRKN mutations. However, the present study showed that the PRKN mutation had little effect on mitochondrial size when comparing dopaminergic neurons between control and PRKN-mutated lines. This result indicates the importance of using labeled dopaminergic neurons for studying dopaminergic neuron-specific phenotypes in PD.

Live cell imaging and flow cytometric analysis of TMRM-stained cells demonstrated that the mitochondrial membrane potential in dopaminergic neuron was markedly lower than that in non-dopaminergic neuron at least in the control lines. This finding implies that mitochondrial function in dopaminergic neurons is correlated with mitochondrial morphology. This observed correlation between mitochondrial morphology and function is supported by many previously published studies. For example, nuclear reprogramming can change oxidative differentiated cells with tubular elongated mitochondria into glycolytic pluripotent stem cells with round-shaped mitochondria [37, 38]. Furthermore, several reports have demonstrated that artificial mitochondrial fragmentation results in impaired mitochondrial function and increased cell death [39-42].

On the other hand, a previous study reported that dopaminergic neurons of the substantia nigra pars compacta have a higher basal rate of mitochondrial oxidative phosphorylation and more complex axons than those of the ventral tegmental area in the mouse [43]. In the study, mitochondrial function in dopaminergic neurons was measured in the whole cell using an extracellular flux analyzer. In our flow cytometric analysis, the decline of the mitochondrial membrane potential in the dopaminergic neurons was milder than that in the soma in live cell imaging. The decline of the mitochondrial membrane potential in the whole cell was probably complemented with a large number of axonal mitochondria in the dopaminergic neurons. In fact, the decline rate of the mitochondrial membrane potential normalized by the mitochondrial mass of the dopaminergic neurons in flow cytometry was similar to that in live cell imaging. Our findings of the small size and low function of mitochondria in the dopaminergic neurons provide a dopaminergic neuron-specific characterization of mitochondria, and would also elucidate one of the possible causes of the high vulnerability of dopaminergic neurons in both familial and sporadic PD.

In the present study, there was no difference in the mitochondrial structure of dopaminergic neurons between control and PRKN-mutated lines under normal conditions using CLEM. However, CCCP treatment induced significant differences in mitochondrial structure and cell death between control and PRKN-mutated dopaminergic neurons. CCCP treatment for $3.5 \mathrm{~h}$ induced the formation of spheroid-shaped mitochondria in control dopaminergic neurons; in contrast, the CCCPinduced formation of spheroid-shaped mitochondria was significantly impaired in PRKN-mutated dopaminergic neurons. Spheroid-shaped mitochondria as a result of mitochondrial uncouplers have been observed in MEF, HEK-293, and HeLa cells, and this spheroid shape is considered to be the morphology of uncoupled mitochondria ready for degradation by lysosomes [17, 18, 34, 35]. CCCP treatment is accompanied by the colocalization of 
mitochondria with lysosomes, irrespective of mitophagy, suggesting that the formation of spheroid-shaped mitochondria may be a novel mechanism of mitochondrial quality control [18]. The present study is the first report of the formation of spheroid-shaped mitochondria in human iPSC-derived dopaminergic neurons. A recent study suggested that spheroid-shaped mitochondrial morphology represents the more stable form of uncoupled mitochondria, and that spheroid-shaped mitochondria undergo mitochondrial degradation [35]. Additionally, previous studies of PRKN-mutated patient iPSC-derived neurons have reported that CCCP treatment for $48 \mathrm{~h}$ induces the accumulation of damaged mitochondria [12, 14]. Thus, it is hypothesized that defects in the formation of spheroid-shaped mitochondria may disturb the degradation of damaged mitochondria in $P R K N$-mutated dopaminergic neurons, resulting in the subsequent cell death of $P R K N$-mutated dopaminergic neurons.

A study by Ding et al. demonstrated that mitochondrial spheroid formation is increased by the knockdown of Parkin in HEK-293 cells [17]. Our study indicated that in dopaminergic neurons the formation of spherical mitochondria was significantly decreased in $P R K N$-mutated lines when compared with control lines. On the other hand, in non-dopaminergic neurons, there was no significant difference in the ratio of the formation of spherical mitochondria between control and PRKN-mutated lines. These differences in the effect of Parkin deficiency on the formation of spherical mitochondria possibly results from the difference in cell types. Taken together, our CLEM analysis of TH-GFP iPSC-derived dopaminergic neurons provided evidence of mitochondrial structural changes before cell death. Furthermore, our study suggests the possibility that defect in mitochondrial spheroid formation contributes to the accumulation of damaged mitochondria and the death of dopaminergic neurons in patients with PRKN mutations. However, the limitation in this study is the small number of TH-GFP iPSC lines derived from two healthy subjects and a patient. Further studies with increased number of TH-GFP iPSC lines and Parkin knockdown/knockout studies using our control iPSC lines are expected to resolve any controversy about the involvement of Parkin expression in mitochondrial spheroid formation.

In conclusion, the current study demonstrated the establishment of control and PRKN-mutated patient TH-GFP iPSC lines for mitochondrial ultrastructural analysis specific to $P R K N$-mutated dopaminergic neurons. CLEM analysis of the TH-GFP iPSC-derived cells revealed the existence of mitochondrial morphology specific to dopaminergic neurons, as well as the insufficient spheroid formation of mitochondria under CCCP treatment in PRKN-mutated dopaminergic neurons. Although the direct relationships between the mitochondrial morphology and the cell death in PRKNmutated dopaminergic neurons have not been clarified in the present study, our in vitro model newly resulted in the discovery of differences in mitochondrial ultrastructural changes between control and $P R K N$-mutated patient under mitochondrial stress. Future studies using our model would reveal the association between defect in mitochondrial spheroid formation and the accumulation of damaged mitochondria and the death of dopaminergic neurons in patients with PRKN mutations. In addition, our workflow-using CLEM analysis of TH-GFP iPSC lines-is also a strong tool for evaluating mitochondrial-derived vesicles, the ER-mitochondria interface, or other organelle impairments in PRKN-mutated patient dopaminergic neurons specifically, under normal and/or pathological conditions, which will help to elucidate the processes leading to the preferential loss of dopaminergic neurons in patients with PRKN mutations.

\section{Supplementary Information}

The online version contains supplementary material available at https://doi. org/10.1186/s13041-021-00771-0.

Additional file 1: Table S1. List of the primers used in this study.

Additional file 2: Figure S1. Characterization of non-dopaminergic neurons differentiated from TH-GFP iPSC lines. Immunofluorescence staining for $\mathrm{TH}, \beta 3$-tubulin, and Nestin identified other neural subtypes $(\beta 3$-tubulin $+\mathrm{TH}-)$ and neural progenitor cells (Nestin $+\mathrm{TH}-)$ among the non-dopaminergic cells derived from TH-GFP iPSC lines. Arrowheads indicate $\beta 3$-tubulin + TH- or Nestin + TH- cells. "PRKN" represents PRKNmutated patient. Scale bar, $20 \mu \mathrm{m}$.

Additional file 3: Figure S2. Flow cytometric analysis of mitochondrial membrane potential in dopaminergic neurons derived from TH-GFP iPSCs. (a) Histograms showed GFP-negative and GFP-positive populations in the TH-GFP iPSC-derived differentiated cells. Data were obtained from three independent experiments. "PRKN" represents PRKN-mutated patient. Values are shown as the mean \pm SEM. (b) TMRM-GFP cytograms (top) represented the intensity of TMRM signal in GFP-negative and -positive populations. Mitotracker DeepRed-GFP cytograms (bottom) represented the intensity of Mitotracker DeepRed signal in GFP-positive and -positive populations. "PRKN" represents PRKN-mutated patient. (c) Quantitative analysis of the MFI of TMRM (left), Mitotracker DeepRed (center), and TMRM normalized with Mitotracker DeepRed (right) in GFP-positive cells relative to GFP-negative cells in the control and PRKN-mutated lines. Data were obtained from three independent experiments. "PRKN" represents PRKN-mutated patient. Values are shown as the mean \pm SEM. Statistical significance was evaluated using the unpaired two-tailed $t$-test. ${ }^{*} P<0.05$. There was no significant difference in the MFI of Mitotracker between control GFP-positive and-negative cells (control center; $P=0.3502$ ). There were no significant differences in the MFI of TMRM and TMRM/Mitotracker between PRKN-mutated GFP-positive and -negative cells (PRKN left; $P=0.5567$, PRKN right; $P=0.1382$ ).

Additional file 4: Figure S3. Mitochondrial membrane potential in iPSCderived cells under CCCP treatment. Mitochondrial membrane potential in dopaminergic and non-dopaminergic neurons derived from control $\mathrm{TH}$ GFP iPSCs under normal conditions and CCCP treatment. CCCP treatment for $3.5 \mathrm{~h}$ rapidly attenuated the fluorescence of TMRM. Scale bar, $5 \mu \mathrm{m}$. 


\section{Acknowledgements}

We thank Prof. Shinya Yamanaka (Kyoto University, Kyoto, Japan) for providing $201 B 7$ iPSCs and Dr. Akitsu Hotta (Kyoto University, Kyoto, Japan) for providing plasmids for genome editing. We also thank Drs. Isei Tanida, Hiroyuki Hioki, Yu-shin Sou, Laxmi Kumar Parajuli, and Kenta Yamauchi for helpful advices, the Laboratory of Cell Biology, Research Support Center, Juntendo University Graduate School of Medicine, and Mitsuko Hosoi for technical assistance. We thank Bronwen Gardner, PhD, from Edanz Group (https://en-author-services. edanzgroup.com/ac) for editing a draft of this manuscript.

\section{Authors' contributions}

$M Y, W A$, and MK designed this study. MY and SK performed the experiments. $\mathrm{MY}, \mathrm{SK}, \mathrm{TS}, \mathrm{KI}, \mathrm{HO}, \mathrm{WA}$, and $\mathrm{MK}$ analyzed and interpreted the data. $\mathrm{HO}$ and $\mathrm{NH}$ contributed to the acquisition of patient samples and discussed the results. MY wrote the first draft of the manuscript. SK, TS, KI, WA, and MK modified the manuscript. All authors approved the final manuscript.

\section{Funding}

This work was funded by the Japan Society for the Promotion of Science (Grant-in-Aid for Early-Career Scientists, $18 K 15466$ to M.Y., and Grants-in-Aid for Scientific Research, 17 K08522 and 20 K07743 to M.K.) and the Ministry of Education, Culture, Sports, Science and Technology of Japan (the Private School Branding Project to M.K., W.A, and N.H, Japan-Supported Programs for the Strategic Research Foundation at Private Universities (S1411007), Scholarship Fund for Women Researchers from The Promotion and Mutual Aid Corporation for Private Schools of Japan (PMAC), the program for women researchers from Juntendo University in 2019-2020, funded by "Initiative for Realizing Diversity in the Research Environment" and the Fostering Physicians in Basic Research for Coping with Advancing Sophistication of Medicine and Medical Care, the Rare/Intractable Disease Project of the Japan, JP19ek0109244 to K.I., N.H., and W.A), and the Japan Agency for Medical Research and Development (Network for Realization Research Centers/Projects of Regenerative Medicine (the Program for Intractable Disease Research Utilizing Disease-specific iPS Cells and the Acceleration Program for Intractable Diseases Research Utilizing Diseasespecific iPS Cells), JP17bm0804003 to W.A. and N.H., and JP20bm0804003 to H.O.), and Institute for Environmental and Gender-specific Medicine and the Research Institute for Diseases of Old Age, Juntendo University.

\section{Availability of data and materials}

The datasets used and/or analysed during the current study are available from the corresponding author on reasonable request.

\section{Ethics approval and consent to participate}

The genetic analysis in the current study was performed under the guidelines of the recombinant DNA experiments committee of Juntendo University (Approval Number 27-3). The use of human iPSCs was approved by the Ethical Committees of Juntendo University School of Medicine (Approval Number 2017032).

\section{Consent for publication}

Not applicable.

\section{Competing interests}

Hideyuki Okano is a founding scientist and a paid scientific advisor of SanbBio Co. Ltd. and K Pharma. Co. Ltd. The other authors declare neither financial nor non-financial competing interests.

\footnotetext{
Author details

${ }^{1}$ Department of Cell Biology and Neuroscience, Juntendo University Graduate School of Medicine, 2-1-1 Hongo, Bunkyo-ku, Tokyo 113-8421, Japan. ${ }^{2}$ Laboratory of Morphology and Image Analysis, Research Support Center, Juntendo University Graduate School of Medicine, Tokyo 113-8421, Japan. ${ }^{3}$ Department of Cellular and Molecular Neuropathology, Juntendo University Graduate School of Medicine, Tokyo 113-8421, Japan. ${ }^{4}$ Center for Genomic and Regenerative Medicine, Juntendo University Graduate School of Medicine, Tokyo 113-8421, Japan. ${ }^{5}$ Department of Neurology, Juntendo University School of Medicine, Tokyo 113-8421, Japan. ${ }^{6}$ Department of Physiology, Keio University School of Medicine, Tokyo 160-8582, Japan. ${ }^{7}$ Advanced Research Institute for Health Sciences, Juntendo University, Bunkyo, Tokyo 113-8421, Japan.
}

Received: 4 September 2020 Accepted: 15 March 2021

Published online: 23 March 2021

\section{References}

1. Abou-Sleiman PM, Muqit MM, Wood NW. Expanding insights of mitochondrial dysfunction in Parkinson's disease. Nat Rev Neurosci. 2006;7(3):207-19.

2. Narendra D, Tanaka A, Suen DF, Youle RJ. Parkin is recruited selectively to impaired mitochondria and promotes their autophagy. J Cell Biol. 2008;183(5):795-803.

3. Scarffe LA, Stevens DA, Dawson VL, Dawson TM. Parkin and PINK1: much more than mitophagy. Trends Neurosci. 2014;37(6):315-24.

4. Dawson TM, Dawson VL. Molecular pathways of neurodegeneration in Parkinson's disease. Science. 2003;302(5646):819-22.

5. Exner N, Lutz AK, Haass C, Winklhofer KF. Mitochondrial dysfunction in Parkinson's disease: molecular mechanisms and pathophysiological consequences. EMBO J. 2012;31(14):3038-62.

6. Goldberg MS, Fleming SM, Palacino JJ, Cepeda C, Lam HA, Bhatnagar A, et al. Parkin-deficient mice exhibit nigrostriatal deficits but not loss of dopaminergic neurons. J Biol Chem. 2003;278(44):43628-35.

7. Palacino JJ, Sagi D, Goldberg MS, Krauss S, Motz C, Wacker M, et al. Mitochondrial dysfunction and oxidative damage in parkin-deficient mice. J Biol Chem. 2004;279(18):18614-22.

8. Von Coelln R, Thomas B, Savitt JM, Lim KL, Sasaki M, Hess EJ, et al. Loss of locus coeruleus neurons and reduced startle in parkin null mice. Proc Natl Acad Sci USA. 2004;101(29):10744-9.

9. Perez FA, Palmiter RD. Parkin-deficient mice are not a robust model of parkinsonism. Proc Natl Acad Sci USA. 2005;102(6):2174-9.

10. Sato S, Chiba T, Nishiyama S, Kakiuchi T, Tsukada H, Hatano T, et al. Decline of striatal dopamine release in parkin-deficient mice shown by ex vivo autoradiography. J Neurosci Res. 2006;84(6):1350-7.

11. Asano T, Koike M, Sakata S, Takeda Y, Nakagawa T, Hatano T, et al. Possible involvement of iron-induced oxidative insults in neurodegeneration. Neurosci Lett. 2015;588:29-35.

12. Imaizumi Y, Okada Y, Akamatsu W, Koike M, Kuzumaki N, Hayakawa H, et al. Mitochondrial dysfunction associated with increased oxidative stress and alpha-synuclein accumulation in PARK2 iPSC-derived neurons and postmortem brain tissue. Mol Brain. 2012;5:35.

13. Suzuki S, Akamatsu W, Kisa F, Sone T, Ishikawa Kl, Kuzumaki N, et al. Efficient induction of dopaminergic neuron differentiation from induced pluripotent stem cells reveals impaired mitophagy in PARK2 neurons. Biochem Biophys Res Commun. 2017;483(1):88-93.

14. Yamaguchi A, Ishikawa KI, Inoshita T, Shiba-Fukushima K, Saiki S, Hatano $\mathrm{T}$, et al. Identifying therapeutic agents for amelioration of mitochondrial clearance disorder in neurons of familial Parkinson disease. Stem Cell Rep. 2020;14(6):1060-75.

15. Ren Y, Jiang H, Hu Z, Fan K, Wang J, Janoschka S, et al. Parkin mutations reduce the complexity of neuronal processes in iPSC-derived human neurons. Stem Cells. 2015;33(1):68-78.

16. Jiang $H$, Ren $Y$, Yuen $E Y$, Zhong $P$, Ghaedi $M, H u$ Z, et al. Parkin controls dopamine utilization in human midbrain dopaminergic neurons derived from induced pluripotent stem cells. Nat Commun. 2012;3:668.

17. Ding WX, Guo F, Ni HM, Bockus A, Manley S, Stolz DB, et al. Parkin and mitofusins reciprocally regulate mitophagy and mitochondrial spheroid formation. J Biol Chem. 2012;287(50):42379-88.

18. Yin XM, Ding WX. The reciprocal roles of PARK2 and mitofusins in mitophagy and mitochondrial spheroid formation. Autophagy. 2013;9(11):1687-92.

19. Sugiura A, McLelland GL, Fon EA, McBride HM. A new pathway for mitochondrial quality control: mitochondrial-derived vesicles. EMBO J. 2014;33(19):2142-56.

20. McLelland GL, Lee SA, McBride HM, Fon EA. Syntaxin-17 delivers PINK1/ parkin-dependent mitochondrial vesicles to the endolysosomal system. J Cell Biol. 2016;214(3):275-91.

21. Gautier CA, Erpapazoglou Z, Mouton-Liger F, Muriel MP, Cormier F, Bigou $\mathrm{S}$, et al. The endoplasmic reticulum-mitochondria interface is perturbed in PARK2 knockout mice and patients with PARK2 mutations. Hum Mol Genet. 2016;25(14):2972-84. 
22. Imaizumi K, Sone T, Ibata K, Fujimori K, Yuzaki M, Akamatsu W, et al. Controlling the regional identity of hPSC-derived neurons to uncover neuronal subtype specificity of neurological disease phenotypes. Stem Cell Rep. 2015;5(6):1010-22.

23. Cui J, Rothstein M, Bennett T, Zhang P, Xia N, Reijo Pera RA. Quantification of dopaminergic neuron differentiation and neurotoxicity via a genetic reporter. Sci Rep. 2016;6:25181.

24. Xia N, Fang F, Zhang P, Cui J, Tep-Cullison C, Hamerley T, et al. A knockin reporter allows purification and characterization of $\mathrm{mDA}$ neurons from heterogeneous populations. Cell Rep. 2017;18(10):2533-46.

25. Calatayud C, Carola G, Fernandez-Carasa I, Valtorta M, Jimenez-Delgado S, Diaz M, et al. CRISPR/Cas9-mediated generation of a tyrosine hydroxylase reporter iPSC line for live imaging and isolation of dopaminergic neurons. Sci Rep. 2019;9(1):6811.

26. Koster AJ, Klumperman J. Electron microscopy in cell biology: integrating structure and function. Nat Rev Mol Cell Biol. 2003;4(Suppl):6-10.

27. Loussert Fonta C, Humbel BM. Correlative microscopy. Arch Biochem Biophys. 2015;581:98-110

28. Hsu PD, Scott DA, Weinstein JA, Ran FA, Konermann S, Agarwala V, et al. DNA targeting specificity of RNA-guided Cas9 nucleases. Nat Biotechnol. 2013;31(9):827-32.

29. Li HL, Fujimoto N, Sasakawa N, Shirai S, Ohkame T, Sakuma T, et al. Precise correction of the dystrophin gene in duchenne muscular dystrophy patient induced pluripotent stem cells by TALEN and CRISPR-Cas9. Stem Cell Rep. 2015;4(1):143-54

30. Takahashi K, Tanabe K, Ohnuki M, Narita M, Ichisaka T, Tomoda K, et al. Induction of pluripotent stem cells from adult human fibroblasts by defined factors. Cell. 2007;131(5):861-72.

31. Wu J, Hunt SD, Xue H, Liu Y, Darabi R. Generation and characterization of a MYF5 reporter human iPS cell line using CRISPR/Cas9 mediated homologous recombination. Sci Rep. 2016:6:18759.

32. Matsumoto T, Fujimori K, Andoh-Noda T, Ando T, Kuzumaki N, Toyoshima $M$, et al. Functional neurons generated from $T$ cell-derived induced pluripotent stem cells for neurological disease modeling. Stem Cell Rep. 2016;6(3):422-35

33. Fujimori K, Matsumoto T, Kisa F, Hattori N, Okano H, Akamatsu W. Escape from pluripotency via inhibition of TGF-beta/BMP and activation of Wnt signaling accelerates differentiation and aging in hPSC progeny cells. Stem Cell Rep. 2017;9(5):1675-91.
34. Ding WX, Li M, Biazik JM, Morgan DG, Guo F, Ni HM, et al. Electron microscopic analysis of a spherical mitochondrial structure. J Biol Chem. 2012:287(50):42373-8.

35. Miyazono Y, Hirashima S, Ishihara N, Kusukawa J, Nakamura KI, Ohta K. Uncoupled mitochondria quickly shorten along their long axis to form indented spheroids, instead of rings, in a fission-independent manner. Sci Rep. 2018;8(1):350.

36. Liang CL, Wang TT, Luby-Phelps K, German DC. Mitochondria mass is low in mouse substantia nigra dopamine neurons: implications for Parkinson's disease. Exp Neurol. 2007;203(2):370-80.

37. Prigione A, Fauler B, Lurz R, Lehrach H, Adjaye J. The senescence-related mitochondrial/oxidative stress pathway is repressed in human induced pluripotent stem cells. Stem Cells. 2010;28(4):721-33.

38. Folmes CD, Nelson TJ, Martinez-Fernandez A, Arrell DK, Lindor JZ, Dzeja $\mathrm{PP}$, et al. Somatic oxidative bioenergetics transitions into pluripotencydependent glycolysis to facilitate nuclear reprogramming. Cell Metab. 2011;14(2):264-71.

39. Frank S, Gaume B, Bergmann-Leitner ES, Leitner WW, Robert EG, Catez F, et al. The role of dynamin-related protein 1 , a mediator of mitochondrial fission, in apoptosis. Dev Cell. 2001;1(4):515-25.

40. Youle RJ, van der Bliek AM. Mitochondrial fission, fusion, and stress. Science. 2012;337(6098):1062-5.

41. Cipolat S, Rudka T, Hartmann D, Costa V, Serneels L, Craessaerts K, et al. Mitochondrial rhomboid PARL regulates cytochrome $c$ release during apoptosis via OPA1-dependent cristae remodeling. Cell. 2006;126(1):163-75.

42. Ramonet D, Perier C, Recasens A, Dehay B, Bove J, Costa V, et al. Optic atrophy 1 mediates mitochondria remodeling and dopaminergic neurodegeneration linked to complex I deficiency. Cell Death Differ. 2013;20(1):77-85

43. Pacelli C, Giguere N, Bourque MJ, Levesque M, Slack RS, Trudeau LE. Elevated mitochondrial bioenergetics and axonal arborization size are key contributors to the vulnerability of dopamine neurons. Curr Biol. 2015;25(18):2349-60.

\section{Publisher's Note}

Springer Nature remains neutral with regard to jurisdictional claims in published maps and institutional affiliations.
Ready to submit your research? Choose BMC and benefit from:

- fast, convenient online submission

- thorough peer review by experienced researchers in your field

- rapid publication on acceptance

- support for research data, including large and complex data types

- gold Open Access which fosters wider collaboration and increased citations

- maximum visibility for your research: over 100M website views per year

At BMC, research is always in progress.

Learn more biomedcentral.com/submissions 